\title{
Decreased expression of nuclear factor 90 correlates with worse outcomes in nasopharyngeal carcinoma
}

\author{
Yong $\mathrm{Li}^{1,2 \#}$, Jianchang $\mathrm{Fu}^{2 \#}, \mathrm{Zi}-\mathrm{Zi} \mathrm{Li}{ }^{3 \#}$, Qiufang $\mathrm{Zhu}^{4}$, Keming Chen ${ }^{2}$, Huixiong $\mathrm{Du}^{4}$, Jun Liu ${ }^{1,2}$, \\ Yingqing $\mathbf{L i}^{1}$
}

${ }^{1}$ State Key Laboratory of Oncology in South China, Collaborative Innovation Center for Cancer Medicine, Guangdong Key Laboratory of Nasopharyngeal Carcinoma Diagnosis and Therapy, Sun Yat-sen University Cancer Center, Guangzhou, China; ${ }^{2}$ Department of Pathology, Sun Yatsen University Cancer Center, Guangzhou, China; ${ }^{3}$ Department of Pathology, the Fifth Affiliated Hospital of Sun Yat-sen University, Zhuhai, China; ${ }^{4}$ Department of Pathology, Zhuhai People's Hospital, Zhuhai, China

Contributions: (I) Conception and design: J Liu, Y Li; (II) Administrative support: K Chen; (III) Provision of study materials or patients: Y Li, J Fu; (IV) Collection and assembly of data: Q Zhu, H Du; (V) Data analysis and interpretation: Y Li, J Fu, ZZ Li; (VI) Manuscript writing: All authors; (VII) Final approval of manuscript: All authors.

"These authors contributed equally to this work.

Correspondence to: Yingqing Li; Jun Liu. State Key Laboratory of Oncology in South China, Collaborative Innovation Center for Cancer Medicine, Guangdong Key Laboratory of Nasopharyngeal Carcinoma Diagnosis and Therapy, Sun Yat-sen University Cancer Center, 651\# Dong Feng Road East, Guangzhou 510060, China. Email: liyingq1@sysucc.org.cn; liujun@sysucc.org.cn.

Background: Nuclear factor 90 (NF90), one of the double-stranded RNA binding proteins (DSRBP), is involved in many cellular biological processes, includes cell proliferation, differentiation, angiogenesis, cell cycle and immunity. However, its role in nasopharyngeal carcinoma (NPC), and the significance of expression in clinics, remains unknown. The aim of this study was to uncover the relationship between the NF90 expression by immunohistochemistry (IHC) and clinical outcomes in NPC patients.

Methods: We retrospectively detected the NF90 expression in 216 NPC specimens by IHC. The cutoff value of NF90 was evaluated using receiver operating characteristic (ROC) curve, the prognostic value of NF90 in NPC was evaluated by Kaplan-Meier and cox multifactor statistical model.

Results: Elevated expression of NF90 was found in 60.2\% (130/216). Positive correlation was found between NF90 expression and clinical stage $(\mathrm{P}=0.018)$, T stage $(\mathrm{P}=0.004)$, and therapy $(\mathrm{P}=0.020)$. Overexpression of NF90 predicted favorable progression-free survival (PFS) $(\mathrm{P}=0.017)$ and overall survival (OS) $(\mathrm{P}<0.001)$ in NPC. Further analysis suggested that the level of NF90 expression was an independent risk factor in NPC prognosis.

Conclusions: These results demonstrate, for the first time, that decreased expression of NF90 is an independent biomarker of worse prognosis for NPC patients, and is a great potential tool for screening the high-risk population for advanced NPC patients.

Keywords: Nasopharyngeal carcinoma (NPC); nuclear factor 90 (NF90); prognosis

Submitted Jun 03, 2020. Accepted for publication Feb 18, 2021.

doi: $10.21037 /$ tcr-20-2252

View this article at: http://dx.doi.org/10.21037/tcr-20-2252

\section{Introduction}

Nasopharyngeal carcinoma (NPC), known as Cantonese Cancer, is one of the most prevalent malignance in Southern China and Southeast Asia $(1,2)$. In endemic regions, such as Southern China, undifferentiated non-keratinizing carcinoma, the major histopathological type, accounts for most cases $(>95 \%)$ and is invariable correlated with EpsteinBarr virus (EBV) infection (3-5). It distinguishes other head and neck carcinomas from epidemiology, symptoms, 
biological markers, and prognostic variables (6). Although living habits, environmental factors, EBV infection, genetics and other oncogenic factors have been reported closely associated with the tumorigenesis of NPC, the precise etiology of NPC is still elusive (6). Radiotherapy is still regarded as the primary and curative therapeutic strategy for NPC, especially for intensity-modulated radiotherapy (IMRT) and chemo-radiotherapy, which partly attribute to disease control and survival improvement $(1,7)$. Nevertheless, distant metastasis often occurs even after curative chemoradiotherapy and contributes to cancer-related mortality. So far, clinical therapeutic design and prediction have been made almost depending on tumor node metastasis (TNM) staging classification. However, it often exhibits completely different prognosis between the same anatomic-based stage NPC patients, which probably attribute to inherent biological heterogeneity of the tumors. Therefore, there would be a pressing need to seek for special molecular biomarkers, which could critically screen high-risk population of recurrences by combining TNM staging system.

Nuclear factor 90 (NF90), known as DRBP76, is a double-stranded RNA binding proteins (DSRBP), which was originally found as a post-transcriptional regulator of interleukin-2 (IL-2) promoter (8). It is conserved in vertebrates, and is transcribed from the interleukin enhancerbinding factor-3 (ILF3) gene (9). It has been suggested that NF90 participates in a series of cellular biological processes, such as cell proliferation, differentiation, angiogenesis, cell cycle and immunity (10-14). In human normal tissues, high expression of NF90 were observed in brain, testis, and thymus, instead, lowly expressed in lung, spleen, skeletal muscle, and liver (12,15-17). Furthermore, NF90 increasingly plays important role in regulating tumorigenesis. Nuclear NF90 overexpression have been reported in many malignancies, including NPC, non-small cell lung cancer, hepatocellular carcinoma, ovarian cancer, breast cancer, cervical cancer and so on $(13,18-22)$. However, the clinical significance of NF90 expression for NPC patients remains unclear and needs to be uncovered. Thus, in this study, NF90 expression was detected by using immunohistochemistry (IHC) staining in two hundred and sixteen NPC biopsies. The association between NF90 expression and patient clinical variables was investigated, whether expression of NF90 would be a prognostic biomarker for patients of NPC. We present the following article in accordance with the REMARK reporting checklist (available at http://dx.doi. org/10.21037/tcr-20-2252).

\section{Methods}

\section{Patients selection}

We retrospectively enrolled 216 NPC patients from the Department of Radiation, Sun Yat-sen University Cancer Center, between October 1998 and April 2012. The definitions of NPC patient tumor histopathology and clinical stage were according to the $8^{\text {th }}$ edition of the TNM Staging system of American Joint Committee on Cancer (AJCC). The recruitment criteria are as follows: (I) newly diagnosed (incident) NPC without receiving any anti-cancer therapy; (II) pathological diagnosis of undifferentiated non-keratinizing carcinoma; (III) paraffin-embedded tissue specimens were available for IHC staining analysis; (IV) complete clinical and followup information. All patients were follow-up ended in June 2018 to ascertain survival status. The study was conducted in accordance with the Declaration of Helsinki (as revised in 2013). The study was approved by the Institute Research Medical Ethics Committee of Sun Yat-sen University Cancer Center (GZR2019-075). No informed consent (written or verbal) was obtained for use of retrospective data from the patients within this study, most of whom were deceased, since this was not deemed necessary by the Ethics Committee, who waived the need for consent. All samples were anonymized.

\section{IHC staining}

IHC staining was conducted according to the guidelines of Envision method. Paraffin sections rehydrated through graded alcohols. Firstly, slides were using 0.3\% hydrogen peroxide for $15 \mathrm{~min}$ blocking the activity of endogenous peroxidase activity, and then were boiled in citrate buffer ( $\mathrm{pH}$ 6.0) for $3 \mathrm{~min}$ in a pressure cooker. Nonspecific binding was blocked with $5 \%$ BSA for $15 \mathrm{~min}$. Subsequently, the slides were incubated with anti-NF90 at a dilution of 1: 600 [a mouse monoclonal antibody to NF90 (21): sc-136197, Santa Cruz] for $50 \mathrm{~min}$ at $37^{\circ} \mathrm{C}$. Lastly, immunostaining detection was followed by incubating the secondary antibody (Envision, Dako, Denmark) for $30 \mathrm{~min}$ at $37^{\circ} \mathrm{C}$, and stained with 3,3-diaminobenzidine (DAB). A normal rabbit IgG was 
used for a negative control.

\section{Immunoreactivity score (IRS) assessment}

The IRS of NF90 expression was performed by two independent pathologists (JF and YL). IRS was based on the staining intensity and scope (0-300 scores). The intensity score was defined: negative [0], weak [1], moderate [2], and strong [3]; and the scope score was defined: counter the percentage in $5 \%$ increments from 0 to $100 \%$ by taking 5 fields in every slide. The detailed scoring criteria were described previously (23). In this study, the slides would be reexamined by the third pathologist if the different scores between observers.

\section{Statistical analysis}

Statistical analysis was carried out with SPSS software (SPSS Standard version 16.0, SPSS, Chicago, IL, USA). The NF90 IRS cutoff value is calculated by the receiver operating characteristic (ROC) method. The correlation of NF90 expression with NPC patient clinicopathologic parameters was evaluated by the Pearson-Chi-square test. A binary logistic regression model was applied for the analysis of variables correlating with NF90 expression. Progressionfree survival (PFS) was defined as the time from receiving anti-cancer therapy to local relapse or distant metastasis. Overall survival (OS) was defined as the time from receiving anti-cancer therapy to cancer-related death. The survival curves of PFS and OS were plotted by Kaplan-Meier method and compared by the log-rank test. Multivariate survival analysis was conducted on all parameters that were found to be significant on univariate analysis using the Cox regression model. All $\mathrm{P}$ value $<0.05$ were regarded as statistically significant.

\section{Results}

\section{Patient characteristics}

Fifty-five females and 161 males were included in our study, aged from 11 to 77 (median 46.0 years). The detailed clinicopathological characteristics of all 216 NPC patients were shown in Table 1.

\section{Definition of NF90 IRS cutoff value}

IRS cutoff value was calculated by using ROC curve analysis, which was defined as approximated to the maximal sensitivity and specificity, and divided NF90 expression patients into two groups. In our study, the sensitivity and specificity for each clinicopathological feature were plotted (Figure 1A,B,C,D,E,F). Survival status was used as the best state variable, the result showed that the IRS 170 was the cutoff value (Figure 1A, $\mathrm{P}=0.005$ ); expression surpass this value was considered high, conversely, low.

\section{Expression of NF90 in NPC}

In this study, NF90 staining of nasopharyngeal cancer tissue was detected in nuclei primarily as described in Figure 2. Using the above criteria, high expression of NF90 was checked in $60.2 \%(130 / 216)$, while low level expression of NF90 was observed in $39.8 \%(86 / 216)$ (Table 1). The relationship between clinicopathologic characteristics and NF90 expression levels of the whole cases was listed in Table 1. The expression of NF90 correlated closely with $\mathrm{T}$ stage $(\mathrm{P}=0.004)$, therapy $(\mathrm{P}=0.020)$ and clinical stage $(\mathrm{P}=0.018)$. No significant association was observed between NF90 expression and gender, age, $\mathrm{N}$ stage, $\mathrm{M}$ stage and relapse.

\section{NF90 expression and clinicopathological characteristics and survival}

In the present study, all of the 216 NPC patients were followed up. The median follow-up period was 70.0 months (2-236 months), with 130 (60.2\%) deceased and 86 still alive. The 5 -year progression-survival rate and overallsurvival rate for the whole cases were $64.8 \%$ and $79.7 \%$, respectively. Then we calculated the different parameters by univariate survival analysis. These results showed that these variables were strongly associated with OS: gender (Table 2, $\mathrm{P}=0.009$ ), age (Table 2, $\mathrm{P}=0.018$ ), clinical stage (Table 2, $\mathrm{P}=0.006$, Figure 3), $\mathrm{T}$ stage (Table 2, $\mathrm{P}=0.03$, Figure 3), $\mathrm{N}$ stage (Table 2, $\mathrm{P}=0.038$, Figure 3), $\mathrm{M}$ stage (Table 2, $\mathrm{P}<0.001$ ), Relapse (Table 2, $\mathrm{P}<0.001$, Figure 3), and NF90 expression (Table 2, $\mathrm{P}<0.001$ ), but insignificant relationship was observed between therapy and OS (Table 2, $\mathrm{P}=0.785$ ). Patients with high expression of NF90 showed more favorable PFS and OS than those with low expression $(\mathrm{P}<0.05$, Figure 3$)$.

In subgroup survival analysis, NF90 expression can distinguish PFS/OS from stage III + IV patients $(\mathrm{P}<0.001$, Figure 4), but not observed in stage I + II stage patients $(\mathrm{P}=0.534$, Figure 4). The same results also were found in $\mathrm{T} 1$ 
Table 1 Correlation between the NF90 expression and clinicopathological variables in 216 patients with NPC

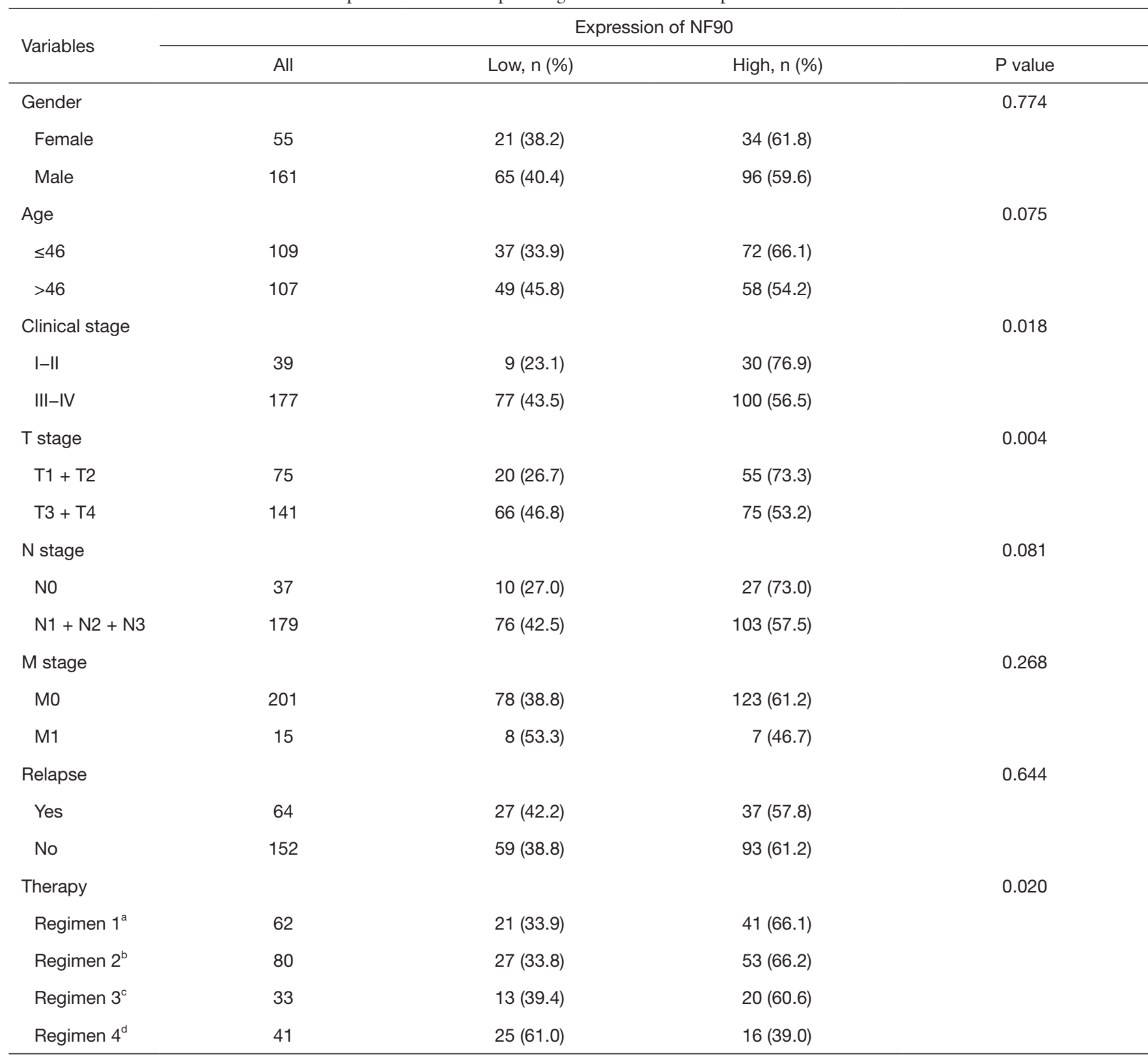

Age: mean age; ${ }^{a}$, radiotherapy; ${ }^{b}$, chemoradiotherapy; ${ }^{c}$, radiotherapy + induce; ${ }^{d}$, chemoradiotherapy + induce. NF90, nuclear factor 90 ; NPC, nasopharyngeal carcinoma.

$+\mathrm{T} 2(\mathrm{P}=0.015$, Figure 4), $\mathrm{T} 3+\mathrm{T} 4(\mathrm{P}<0.001$, Figure 4) and $\mathrm{N}$ positive patients $(\mathrm{P}<0.001$, Figure 4$)$.

\section{Independent prognostic factors for NPC patients}

Univariate analysis using Cox's proportional hazard model found that these variables correlated significantly with OS: gender, age, $\mathrm{T}$ stage, $\mathrm{N}$ stage, $\mathrm{M}$ stage and NF90 expression. Further, we observed that the significant and independent prognostic factors were age, gender, $M$ stage and NF90 expression (i.e., $\mathrm{OS})(\mathrm{RR}=1.770, \mathrm{P}=0.031$; $\mathrm{RR}=2.342, \mathrm{P}=0.028 ; \mathrm{RR}=2.833, \mathrm{P}=0.004 ; \mathrm{RR}=0.288$, 


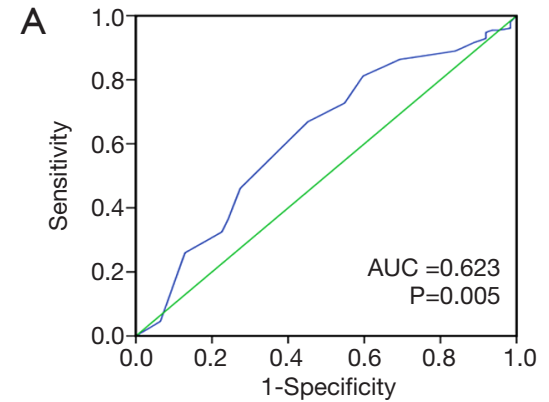

D

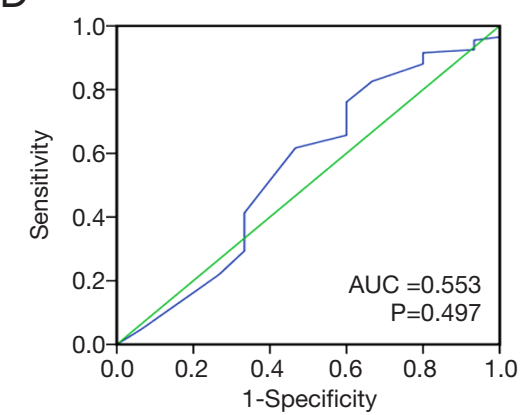

B

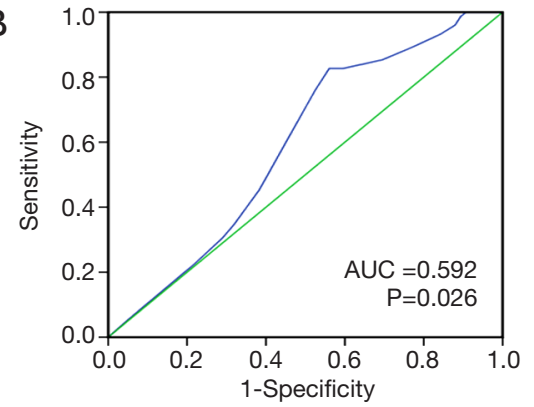

E

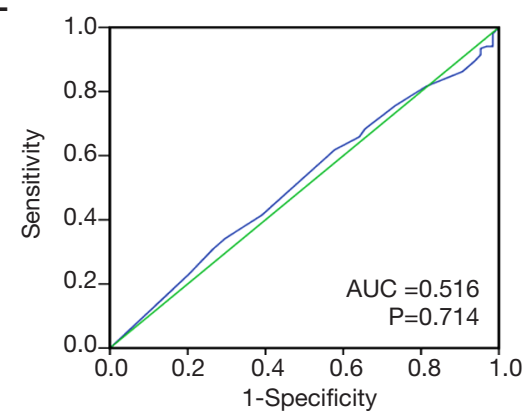

C

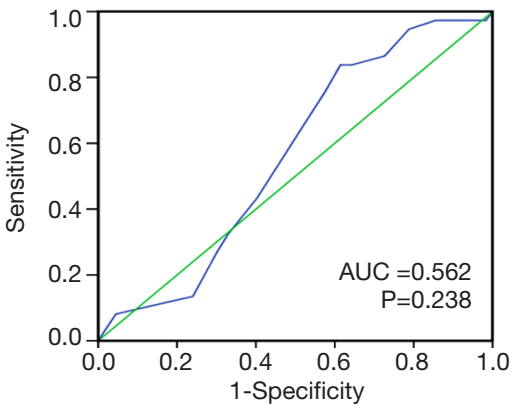

$\mathrm{F}$

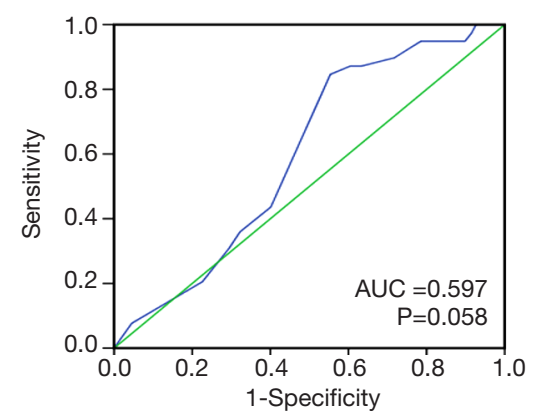

Figure 1 ROC curve analysis was employed to determine the cut-off value for high NF90 expression in NPC. The sensitivity and specificity for each outcome were plotted: (A) survival outcome, (B) T stage, (C) N stage, (D) M stage, (E) relapse, (F) clinical stage. ROC, receiver operating characteristic; NF90, nuclear factor 90; NPC, nasopharyngeal carcinoma; AUC, area under the ROC curve.

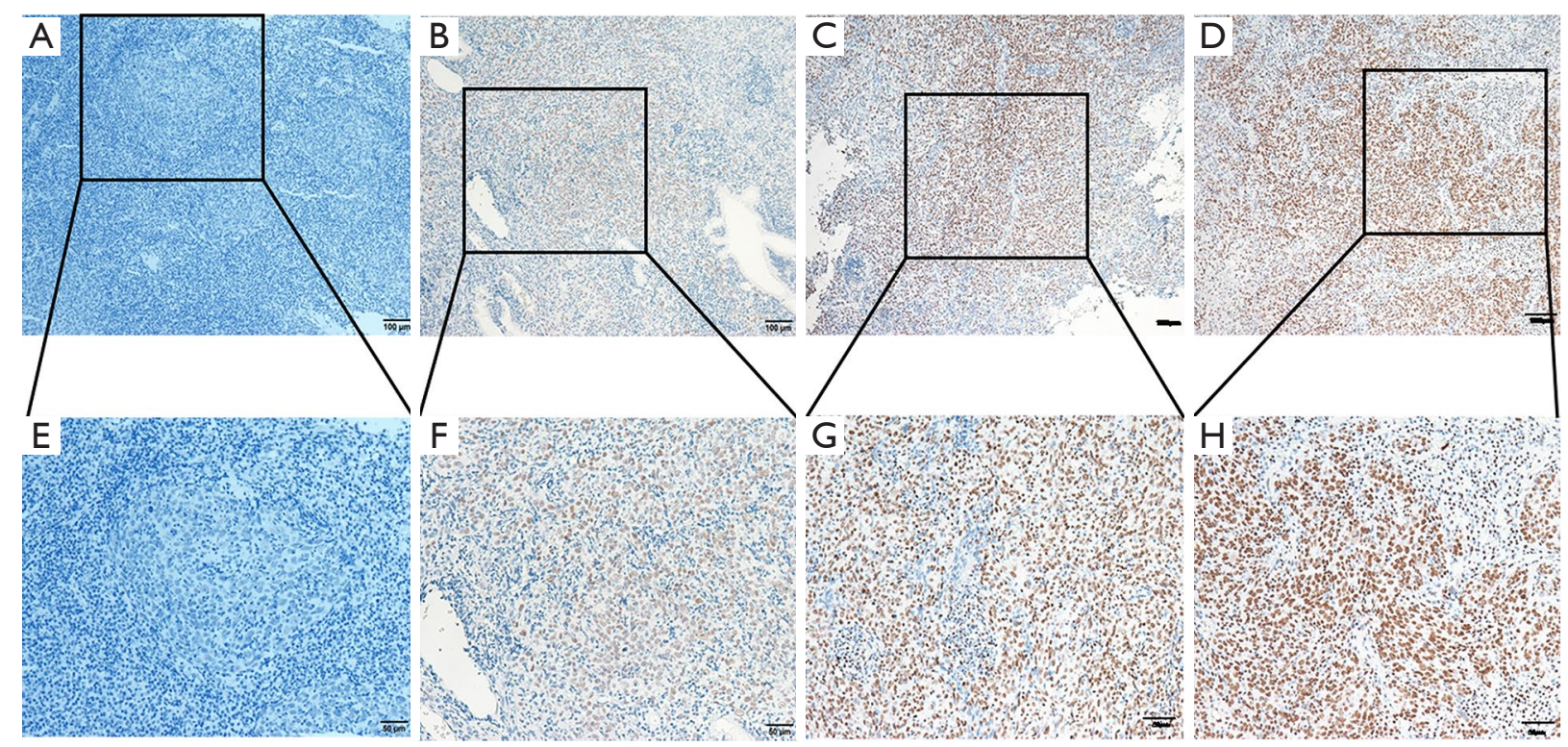

Figure 2 IHC staining of NF90 expression in NPC tissues: negative expression $(A)(\times 10),(E)(\times 20)$; low expression $(B)(\times 10),(F)(\times 20)$; moderate expression (C) (×10), (G) (×20); strong expression (D) $(\times 10),(H)(\times 20)$. NF90, nuclear factor 90; NPC, nasopharyngeal carcinoma. 
Table 2 Univariate survival analysis of different prognostic factors in 216 patients with NPC

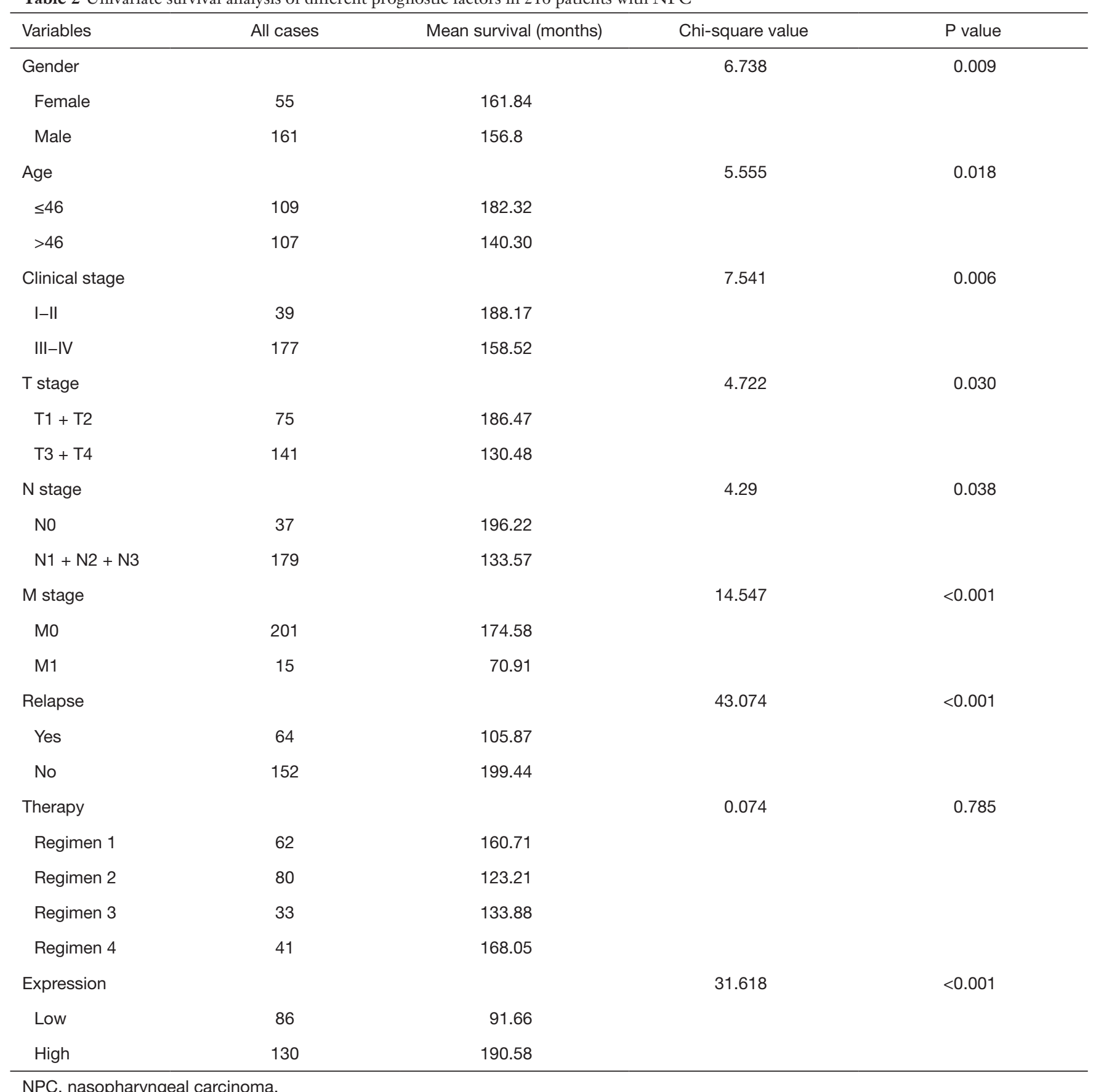

$\mathrm{P}<0.001$ respectively, Table 3).

\section{Discussion}

NF90, also known as DRBP76 or NFAR1, has been reported functions in many cellular processes, including immunity, cell proliferation, differentiation, angiogenesis, cell cycle regulation, and DNA-break repair (10-14,24,25). Up to now, many studies have also demonstrated NF90 participation in oncogenic processes, including NPC, nonsmall cell lung cancer, hepatocellular carcinoma, ovarian cancer, breast cancer, cervical cancer and so on $(13,18-22)$. 
A

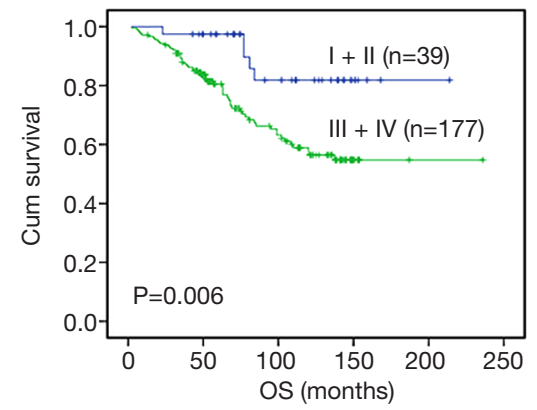

D

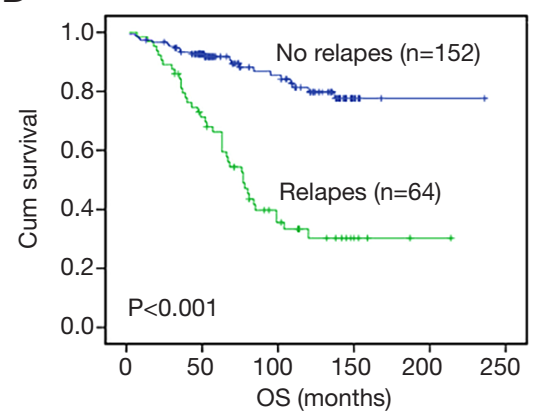

B

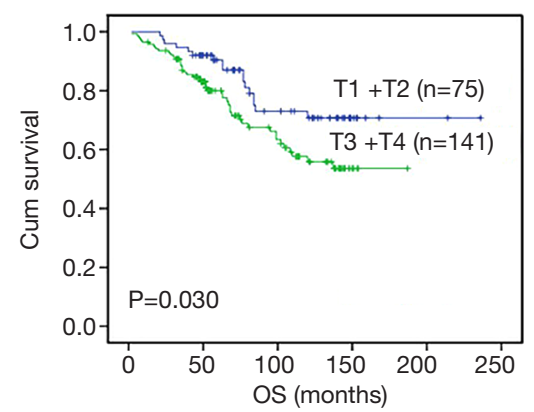

E

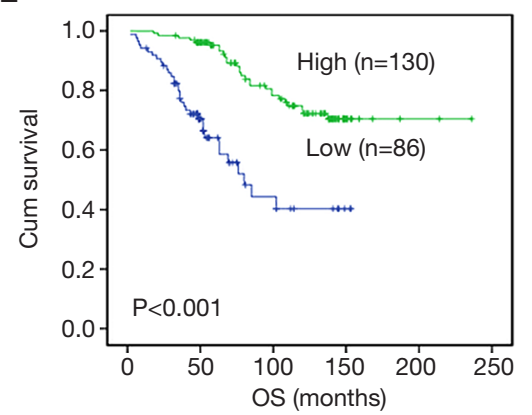

C

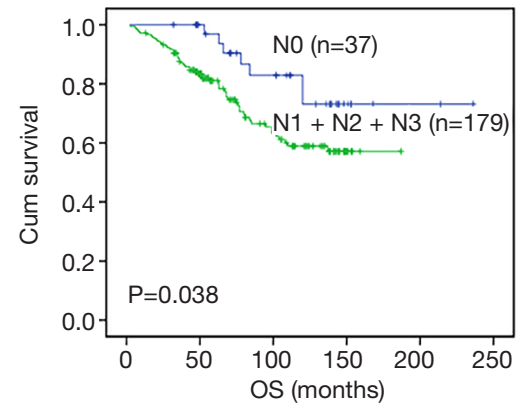

$\mathrm{F}$

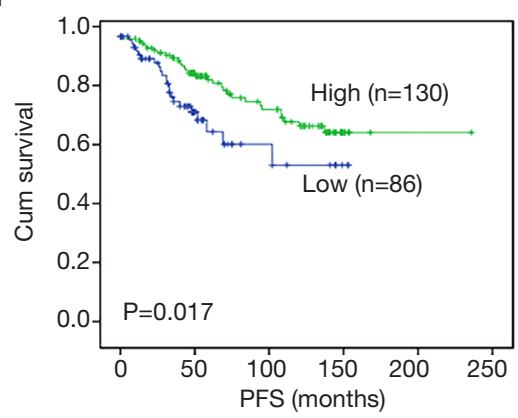

Figure 3 Different prognostic factors for survival outcome in 216 patients with NPC were plotted: (A) clinical stage, (B) $\mathrm{T}$ stage, (C) N stage, (D) relapse, (E) NF90 expression (OS), (F) NF90 expression (PFS). NPC, nasopharyngeal carcinoma; NF90, nuclear factor 90; OS, overall survival; PFS, progression-free survival.
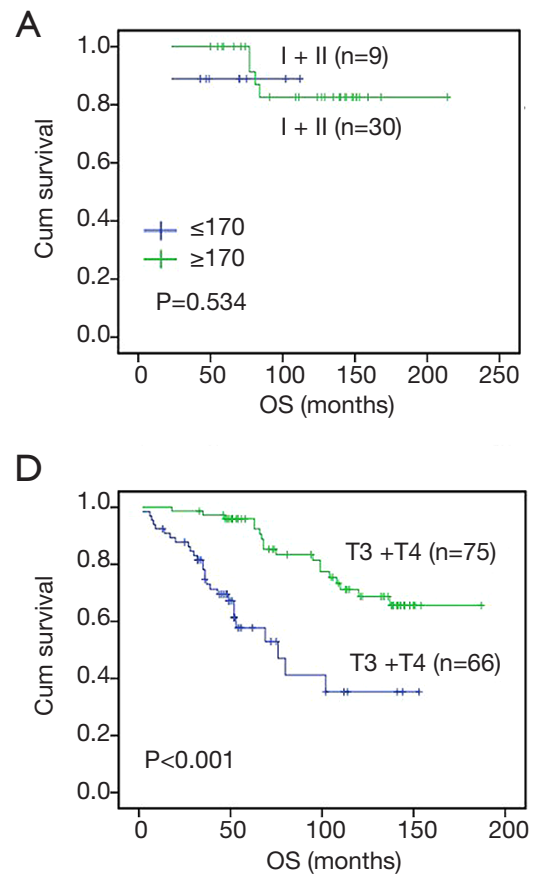
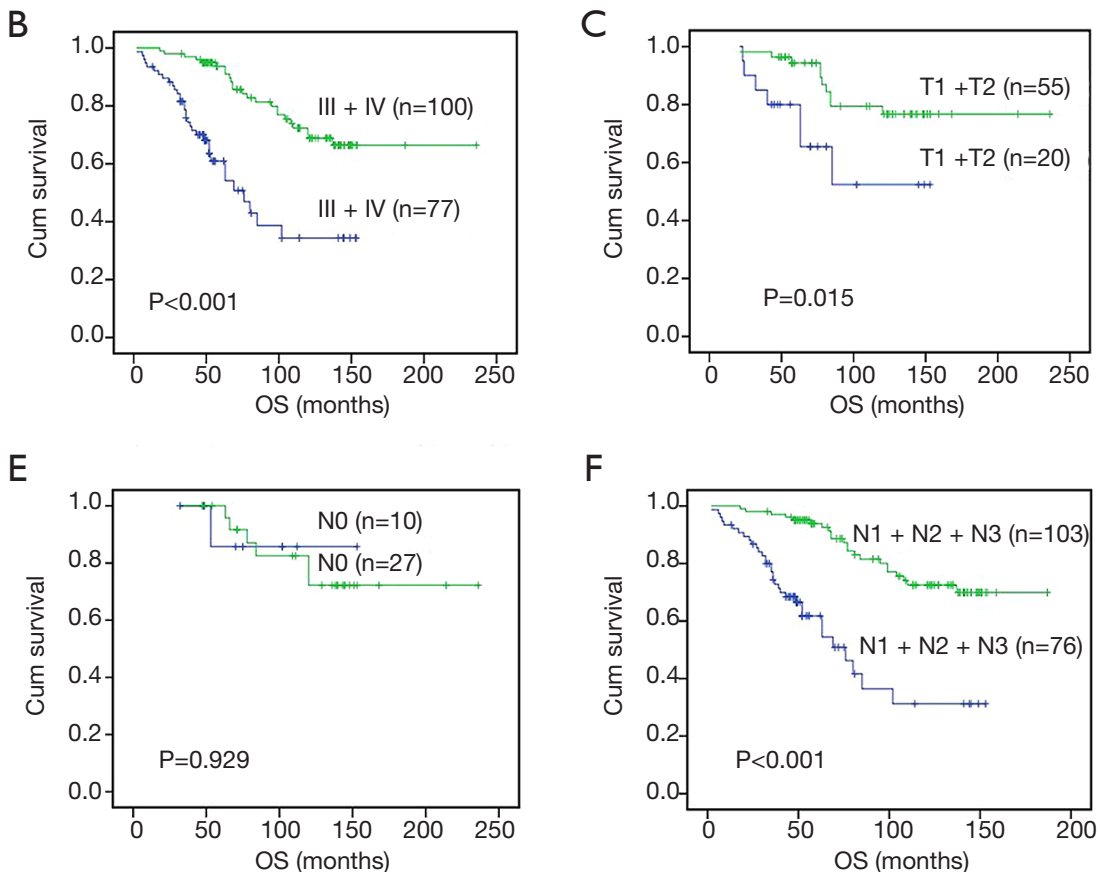

Figure 4 Kaplan-Meier survival analysis of NF90 expression in subsets of NPC patients with different clinical stage and T/N stage. (A) Clinical stage I + II, (B) clinical stage III + IV, (C) T1 + T2, (D) T3 + T4, (E) N0, (F) N1 + N2 + N3. NF90, nuclear factor 90; NPC, nasopharyngeal carcinoma; OS, overall survival. 
Table 3 Results of univariate and multivariate survival analyses for OS according to the Cox Regression Model

\begin{tabular}{|c|c|c|c|c|c|c|}
\hline Variables & \multicolumn{3}{|c|}{ Univariate survival analysis } & \multicolumn{3}{|c|}{ Multivariate survival analysis } \\
\hline Age (>46 vs. $\leq 46)$ & 1.818 & $1.097-3.015$ & 0.020 & 1.770 & $1.053-2.974$ & 0.031 \\
\hline Gender (male vs. female) & 2.575 & $1.225-5.414$ & 0.013 & 2.342 & $1.096-5.004$ & 0.028 \\
\hline T stage $(\mathrm{T} 3+\mathrm{T} 4$ vs. $\mathrm{T} 1+\mathrm{T} 2)$ & 1.840 & $1.051-3.220$ & 0.033 & 1.615 & $0.909-2.867$ & 0.102 \\
\hline M stage (M1 vs. M0) & 3.471 & $1.753-6.872$ & $<0.001$ & 2.833 & $1.382-5.804$ & 0.004 \\
\hline Therapy (regimen $4 / 3 / 2$ vs. regimen 1 ) & 0.804 & $0.479-1.350$ & 0.409 & - & - & - \\
\hline NF90 expression (high vs. low) & 0.247 & $0.146-0.416$ & $<0.001$ & 0.288 & $0.168-0.492$ & $<0.001$ \\
\hline
\end{tabular}

OS, overall survival; HR, hazard ratio; $\mathrm{Cl}$, confidential interval; NF90, nuclear factor 90 .

However, the clinical status of NF90 and potential prognosis in NPC patients remains unknown. In this study, we found NF90 expression was predominantly in the nucleus and the upregulation of NF90 in NPC by using IHC staining method. These results were consistent with those previous reports in lung cancer, hepatocellular carcinoma, etc. $(13,18-22)$. Interestingly, in human normal tissues, high expression of NF90 protein was observed in undifferentiated tissues. But in our enrolled NPC specimens, the histopathological type was almost undifferentiated non-keratinizing carcinoma, also highly expressed NF90. This phenomenon suggests that NF90 may involve in regulation cellular differentiation of NPC, the mechanism needed to be further explored.

To verify the significance of NF90 protein in NPC, we choose an objective cut-point by ROC curve analysis. Further analysis showed that high level of NF90 expression was correlated with clinical stage, advanced $\mathrm{T}$ stage and therapy. More importantly, elevated NF90 expression was a strong and independent parameter in predicting OS advantage by log-rank test and Cox regression method. Moreover, in subgroup analysis, NF90 expression could distinguish high risk of disease progression from advanced clinical stage NPC patients, especially for stage III and IV. Taken together, in our present study, these findings suggest that high expression of NF90 may be a protective factor from NPC progression and play a critical role of suppressor in NPC tumorigenesis. However, in hepatocellular carcinoma, repression of NF90 retards the cell-cycle progression, restricts cell proliferation, and sensitizes cancer cells to cyclin-dependent kinase (CDK) inhibitor, which attributes to NF90 bounding to CyclinE1 mRNA (26).
And in cervical cancer, NF90 promotes tumorigenic capacity via PI3K/Akt signaling pathway in vivo (22). In breast cancer, nuclear up-regulation of NF90 was closely correlated with higher histopathologic grades, but not with OS (21). These findings imply that over-expression of NF90 may be associated with progression in certain cancers, which was inconsistent with these results of present study in NPC. Nevertheless, previous study has found NF90 was involved in host anti-viral mechanism, which exerts its antiviral activity through modulation of PKR phosphorylation (27). Besides, NF90 has been found to be dispensable for IL-2 upregulation via one of the protein kinase $\mathrm{C}$ (PKC) activators stimulation, which was capable of driving the expansion of activated $\mathrm{T}$ cell population, especially for CD8+ T cells $(28,29)$. As we know, NPC, especially for the type of undifferentiated non-keratinizing carcinoma, is closely associated with the EBV infection. And previous clinical studies have reported plasma EBV DNA level independently predict clinical outcomes for NPC patients (30). Thus, we speculate that high expression of NF90 in NPC could exert its dual effects by anti-virus and $\mathrm{T}$ cell activation, and suppose that NF90 is likely to be one of the important immunity activator involved in NPC development. Further studies are required to precisely demonstrate the potential function of NF90 in NPC pathogenesis.

Radiotherapy has been well-known as the optimal treatment for NPC patients, and TNM staging has been recognized as the most precise prognostic factor for clinic therapeutic decision. Even though the patients with the same TNM stage received the same treatments, their outcomes differed widely. Hence, it is urgent need for 
effective predictors of radiation to make individualized therapy. In this study, NF90 expression was found to be closely associated with PFS and OS in NPC patients. Moreover, NF90 high expression for the same stage NPC patients, were at lower probability of recurrence or metastasis.

There are also some limitations in our study. Firstly, it is a retrospective study, required further prospective studies to verify these findings. Besides, the cases enrolled are only from one single institution might cause choosing bias. Lastly, the specimens were all biopsied may not enough for representing the gross tumors.

In conclusion, NF90, the expression examined by IHC, may serve as a new prognostic biomarker for NPC patients, and a great potential tool for screening the high-risk population for advanced NPC patients.

\section{Acknowledgments}

Funding: This study was supported by the Youth Foundation of National Natural Science Foundation of China (81702755).

\section{Footnote}

Reporting Checklist: The authors have completed the REMARK reporting checklist. Available at http://dx.doi. org/10.21037/tcr-20-2252

Data Sharing Statement: Available at http://dx.doi. org/10.21037/tcr-20-2252

Peer Review File: Available at http://dx.doi.org/10.21037/tcr20-2252

Conflicts of Interest: All authors have completed the ICMJE uniform disclosure form (available at http://dx.doi. org/10.21037/tcr-20-2252). The authors have no conflicts of interest to declare.

Ethical Statement: The authors are accountable for all aspects of the work in ensuring that questions related to the accuracy or integrity of any part of the work are appropriately investigated and resolved. The study was conducted in accordance with the Declaration of Helsinki (as revised in 2013). The study was approved by the Institute Research Medical Ethics Committee of Sun Yat-sen University Cancer Center (GZR2019-075). No informed consent (written or verbal) was obtained for use of retrospective data from the patients within this study, most of whom were deceased, since this was not deemed necessary by the Ethics Committee, who waived the need for consent.

Open Access Statement: This is an Open Access article distributed in accordance with the Creative Commons Attribution-NonCommercial-NoDerivs 4.0 International License (CC BY-NC-ND 4.0), which permits the noncommercial replication and distribution of the article with the strict proviso that no changes or edits are made and the original work is properly cited (including links to both the formal publication through the relevant DOI and the license). See: https://creativecommons.org/licenses/by-nc-nd/4.0/.

\section{References}

1. Chua MLK, Wee JTS, Hui EP, et al. Nasopharyngeal carcinoma. Lancet 2016;387:1012-24.

2. Torre LA, Siegel RL, Ward EM, et al. Global cancer incidence and mortality rates and trends--an update. Cancer Epidemiol Biomarkers Prev 2016;25:16-27.

3. Pathmanathan R, Prasad U, Sadler R, et al. Clonal proliferations of cells infected with Epstein-Barr virus in preinvasive lesions related to nasopharyngeal carcinoma. N Engl J Med 1995;333:693-8.

4. Sham JS, Wei W, Ng MH, et al. Nasopharyngeal carcinoma research in Hong Kong in the past decade. Chin Med J (Engl) 1997;110:826-31.

5. Wei WI, Sham JS. Nasopharyngeal carcinoma. Lancet 2005;365:2041-54.

6. Thompson L. Nasopharyngeal carcinoma. Ear Nose Throat J 2005;84:404-5.

7. Zhang L, Huang Y, Hong S, et al. Gemcitabine plus cisplatin versus fluorouracil plus cisplatin in recurrent or metastatic nasopharyngeal carcinoma: a multicentre, randomised, open-label, phase 3 trial. Lancet 2016;388:1883-92.

8. Corthésy B, Kao PN. Purification by DNA affinity chromatography of two polypeptides that contact the NFAT DNA binding site in the interleukin 2 promoter. J Biol Chem 1994;269:20682-90.

9. Duchange N, Pidoux J, Camus E, et al. Alternative splicing in the human interleukin enhancer binding factor 3 (ILF3) gene. Gene 2000;261:345-53.

10. Shim J, Lim H, Yates JR, et al. Nuclear export of NF90 is required for interleukin-2 mRNA stabilization. Mol Cell 
2002;10:1331-44.

11. Vumbaca F, Phoenix KN, Rodriguez-Pinto D, et al. Double-stranded RNA-binding protein regulates vascular endothelial growth factor mRNA stability, translation, and breast cancer angiogenesis. Mol Cell Biol 2008;28:772-83.

12. Shi L, Zhao G, Qiu D, et al. NF90 regulates cell cycle exit and terminal myogenic differentiation by direct binding to the 3 '-untranslated region of MyoD and p21WAF1/CIP1 mRNAs. J Biol Chem 2005;280:18981-9.

13. Fung LF, Lo AK, Yuen PW, et al. Differential gene expression in nasopharyngeal carcinoma cells. Life Sci 2000;67:923-36.

14. Matsumoto-Taniura N, Pirollet F, Monroe R, et al. Identification of novel $\mathrm{M}$ phase phosphoproteins by expression cloning. Mol Biol Cell 1996;7:1455-69.

15. Saunders LR, Perkins DJ, Balachandran S, et al. Characterization of two evolutionarily conserved, alternatively spliced nuclear phosphoproteins, NFAR1 and -2 , that function in mRNA processing and interact with the double-stranded RNA-dependent protein kinase, PKR. J Biol Chem 2001;276:32300-12.

16. Sakamoto S, Morisawa K, Ota K, et al. A binding protein to the DNase I hypersensitive site II in HLADR alpha gene was identified as NF90. Biochemistry 1999;38:3355-61.

17. Zhao G, Shi L, Qiu D, et al. NF45/ILF2 tissue expression, promoter analysis, and interleukin-2 transactivating function. Exp Cell Res 2005;305:312-23.

18. Guo NL, Wan YW, Tosun K, et al. Confirmation of gene expression-based prediction of survival in non-small cell lung cancer. Clin Cancer Res 2008;14:8213-20.

19. Sakamoto S, Aoki K, Higuchi T, et al. The NF90-NF45 complex functions as a negative regulator in the microRNA processing pathway. Mol Cell Biol 2009;29:3754-69.

20. Guo Y, Fu P, Zhu H, et al. Correlations among ERCC1, XPB, UBE2I, EGF, TAL2 and ILF3 revealed by gene signatures of histological subtypes of patients with

Cite this article as: $\mathrm{Li} \mathrm{Y,} \mathrm{Fu} \mathrm{J,} \mathrm{Li} \mathrm{ZZ,} \mathrm{Zhu} \mathrm{Q,} \mathrm{Chen} \mathrm{K,} \mathrm{Du} \mathrm{H,}$ Liu J, Li Y. Decreased expression of nuclear factor 90 correlates with worse outcomes in nasopharyngeal carcinoma. Transl Cancer Res 2021;10(5):2318-2327. doi: 10.21037/tcr-20-2252 epithelial ovarian cancer. Oncol Rep 2012;27:286-92.

21. $\mathrm{Hu} \mathrm{Q}, \mathrm{Lu} \mathrm{YY,} \mathrm{Noh} \mathrm{H,} \mathrm{et} \mathrm{al.} \mathrm{Interleukin} \mathrm{enhancer-binding}$ factor 3 promotes breast tumor progression by regulating sustained urokinase-type plasminogen activator expression. Oncogene 2013;32:3933-43.

22. Zhang $W$, Xiong Z, Wei $T$, et al. Nuclear factor 90 promotes angiogenesis by regulating HIF-1alpha/VEGF-A expression through the PI3K/Akt signaling pathway in human cervical cancer. Cell Death Dis 2018;9:276.

23. Chen J, Li S, Xiao Y, et al. p53R2 as a novel prognostic biomarker in nasopharyngeal carcinoma. BMC Cancer 2017;17:846.

24. Wu TH, Shi L, Adrian J, et al. NF90/ILF3 is a transcription factor that promotes proliferation over differentiation by hierarchical regulation in K562 erythroleukemia cells. PLoS One 2018;13:e0193126.

25. Shamanna RA, Hoque M, Lewis-Antes A, et al. The NF90/NF45 complex participates in DNA break repair via nonhomologous end joining. Mol Cell Biol 2011;31:4832-43.

26. Jiang W, Huang H, Ding L, et al. Regulation of cell cycle of hepatocellular carcinoma by NF90 through modulation of cyclin E1 mRNA stability. Oncogene 2015;34:4460-70.

27. Wen X, Huang X, Mok BW, et al. NF90 exerts antiviral activity through regulation of $\mathrm{PKR}$ phosphorylation and stress granules in infected cells. J Immunol 2014;192:3753-64.

28. Zhu P, Jiang W, Cao L, et al. IL-2 mRNA stabilization upon PMA stimulation is dependent on NF90-Ser647 phosphorylation by protein kinase CbetaI. J Immunol 2010;185:5140-9.

29. Spolski R, Li P, Leonard WJ. Biology and regulation of IL-2: from molecular mechanisms to human therapy. Nat Rev Immunol 2018;18:648-59.

30. Wang WY, Twu CW, Chen HH, et al. Long-term survival analysis of nasopharyngeal carcinoma by plasma EpsteinBarr virus DNA levels. Cancer 2013;119:963-70. 